\title{
Flow Around an Autonomous Underwater Vehicle With Bio-Inspired Coating
}

Scott Watkins, BS ${ }^{1}$, Jose Montoya-Segnini, $\mathrm{MS}^{2}$, Burak Aksak, $\mathrm{PhD}^{1}$, Serdar Gorumlu, BS ${ }^{1}$, Amirkhosro Kazemi, $\mathrm{PhD}^{3}$, Oscar Curet, $\mathrm{PhD}^{3}$, Humberto Bocanegra Evans, $\mathrm{PhD}^{1}$, Leonardo P. Chamorro, $\mathrm{PhD}^{4}$, Gerardo Carbajal, $\mathrm{PhD}^{5}$ Luciano Castillo, $\mathrm{PhD}^{2}$

\author{
${ }^{1}$ Texas Tech University, USA, Scott.watkins@ttu.edu \\ 2Purdue University, USA, montoyas@purdue.edu \\ ${ }^{3}$ Florida Atlantic University, USA, ocuret@fau.edu \\ ${ }^{4}$ University of Illinois at Urbana-Champaign, USA \\ ${ }^{5}$ Universidad del Turabo, USA
}

\begin{abstract}
Flow separation is a major factor in the form drag experienced by a moving object. In particular, suppressing or reducing flow separation is critical in the reduction of energy expenditure of autonomous underwater vehicles. Previous research suggests that bio-inspired micro-fibrillar structures are capable of reducing the boundary layer separation in a turbulent flow. Here, we present laboratory measurements using particle tracking velocimetry near the wall of two submersible vessel models: one coated with an array of micro-fibers and a second one with smooth walls. The flow around the vessels was enticed by the ordered micro-fibers to retain higher velocities near the wall of the vessel. The experiments suggest that separation of the flow may be reduced by the use of the bio-inspired micro-fibers.

Keywords-Bio-inspired, Drag reduction, Flow Separation,
\end{abstract} $U A V, P T V$

\section{NOMENCLATURE}

$\begin{array}{ll}\operatorname{Re} & \text { Reynolds number } \\ \mathrm{U} / U_{\infty}: & \text { Normalized streamwise velocity } \\ \mathrm{V} / U_{\infty}: & \text { Normalized streamwise velocity } \\ \mathrm{X} & \text { Streamwise position, mm } \\ \mathrm{Y} & \text { Vertical position, mm }\end{array}$

\section{INTRODUCTION}

Autonomous underwater vehicles (AUVs) are capable of extending the exploration of oceans and other water ways by means of increased depth, mission length, and access to dangerous environments. Since these vessels lack the requirement of supporting human life and habitable volume they can be much smaller, and allow for longer missions. This also allows the vessels to reach far deeper than any manned craft could reach safely and may also enter more hostile environments. This small size however, comes at the cost of smaller power storage capacity with which to support long term missions. In order to increase the length of possible missions there are two basic options: increase the total energy storage, by means of larger vessels or more compact power supplies; or reduce the amount of energy needed. For this study, the focus will be on the latter. A bio-inspired microfiber coating was tested for the ability to reduce flow separation around a small submerged vessel in a laminar flow.

While previous works focused on the riblets and details of the shark denticles the current work focuses on a more macroscale interpretation of the denticles in order to reduce the complexity of production. This reduction in complexity should increase the ability for mass production of the micro scale structures. Simplification of the micro fiber structure due to this process inherently creates different conditions than are present with natural shark denticles. It is unknown how these differences will affect the ability of the fibers to reduce the drag or flow separation on the surface presented.

\section{BACKGROUND}

The parasitic drag on vehicles that move through fluid media is caused by the separation of flow from the body of the vessel. To mitigate this separation, streamlined shapes are often used. These shapes are effective at reducing the drag on a vehicle but cannot always be designed for maximum effectiveness and do not eliminate separation entirely [1]. To further eliminate drag on a vehicle, surface coatings and surface modifications can be added. These surface coatings often present forms that would be intuitively predicted to increase friction drag, pressure drag, or both. While streamlining tends to eliminate sharp corners and generally makes a surface smoother, these coatings tend to add sharp edges to the surface, though these changes are often on a smaller scale than streamlining.

Efforts to create these surfaces have produced many solutions. It has become widely known that the use of roughness on a surface will increase turbulence and this increase in fluid mixing can counteract the separation of a laminar flow [2]. However, experiments with turbulent flow have found that roughness increases the size of the separation bubble. It is suggested that the increase in the size of the turbulent boundary layer outweighs the benefits from the increased turbulent mixing. The study further points out that roughness characterization by means of height alone is not enough. Another study used a less random design to achieve manipulation of the flow over an airfoil [3]. Using a single line of dimples in the surface of the airfoil, as inspired by the dimples of a golf ball, the separation of the flow over the airfoil was delayed. These dimples, when placed at a specific location, created a suction that assisted in keeping the flow attached to the surface. This study also evaluated raised bumps 
and, while there was a reduction in separation, there was also a reduction in lift.

Long grooves in the surface are a common experimental interest in the reduction of separation. Using transverse grooves of square and sinusoidal shapes separation was reduced under an adverse pressure gradient [4]. The study found that the sinusoidal shaped grooves where more effective than the square shaped grooves finding that the former were more effective at creating mixing in the boundary layer. The grooves also housed vortices that were capable of allowing flow to slip over the surfaces. Known as riblets, grooves aligned with the direction of flow are also used to influence the separation of flow from a surface. The evolution of scallop shells revealed a relationship between the spacing of riblets and drag reduction benefits [5]. Numerical investigation of riblets revealed that the reduction of drag caused by riblets is related to the interference with vortex structures and turbulence near the wall [6]. The study mentions that the presence of the riblets allow for stability in low velocity flow near the wall and a reduction in stress.

Mako shark denticles have been of great interest to researchers for some time due to the shark's speed and the interesting mechanics of the denticles themselves. A study that produced 3D printed models of shark denticles were able to gain drag reduction; however, found increased drag when speeds were increased [7]. This experiment also tested the manufactured denticles on a surface allowed to curve and found greater drag reduction. It is worth noting that the artificial denticles were not able to overlap each other as natural shark denticles do. A simplification of the shark denticles has also been tested and applied to the form of an airfoil [8]. The simplified denticles lacked riblets and overlapping. They were also axisymmetric which would eliminate another of the methods that was described for the reduction of backflow. [4] The results of the experiment found a delay in separation of flow but does not attribute this to the production of turbulence by the coating. It is this coating that will be tested in the present study.

The separation of flow from a body in a fluid is known to be caused by back flow or stagnation of fluid at a surface. If backflow occurs on a surface, the incoming flow will be directed away from the surface. This back flow is caused by the presence of an adverse pressure gradient, which is commonly produced when flow enters a region with an increasing volume, as is found behind any object moving through a fluid. The expectation of this study is the resistance of backflow or stagnation in a flow diverging region at the back of UAVs where an adverse pressure gradient is expected.

\section{EXPERIMENTAL SETUP}

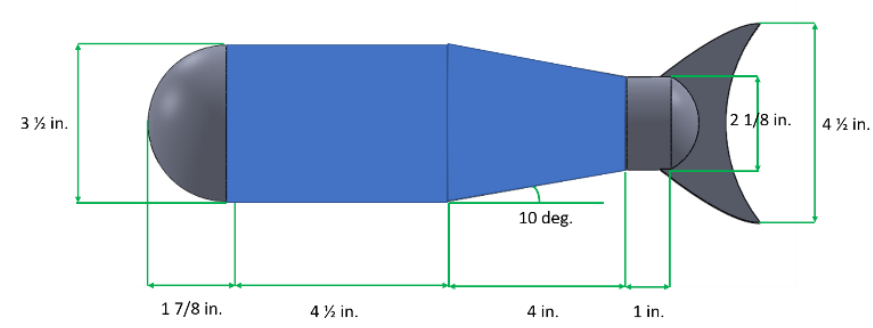

Fig. 1: Model Dimensions. The blue sections represent the sections covered with either the micro-fiber coating or smooth coating.

To test the effect of the micro-fibers on the flow around a submerged vehicle, two small cylindrical vessels were built and coated such that one vessel had a micro-fiber coating and the other had a smooth coating of the same material. The dimensions of the models are presented in Fig. 1 and present a length-based Reynold's number of $\mathrm{Re} \approx 45,000$ based on the approximate freestream flow velocity of $14 \mathrm{~cm} / \mathrm{s}$. The back end of the vessels is tapered at an angle of 10 degrees from the flow direction. This taper introduces an adverse pressure gradient that may be utilized to induce flow separation. If flow separation occurs on the smooth coated vessel and is either absent or occurs later on the micro-fiber coated vessel, then the micro-fiber coating can be said to reduce the separation of flow. The coating was only applied on the body of the vessel and the tapered section, marked by the blue sections in Fig. 1, due to geometrical limitations. These vessels were fitted with a tail for use in other experiments that is rotated $90^{\circ}$ as to not interfere with the flow measurement of this experiment. The vessels were mounted inside an open top water tunnel using a mounting bar on the top of the vessel so that measurements

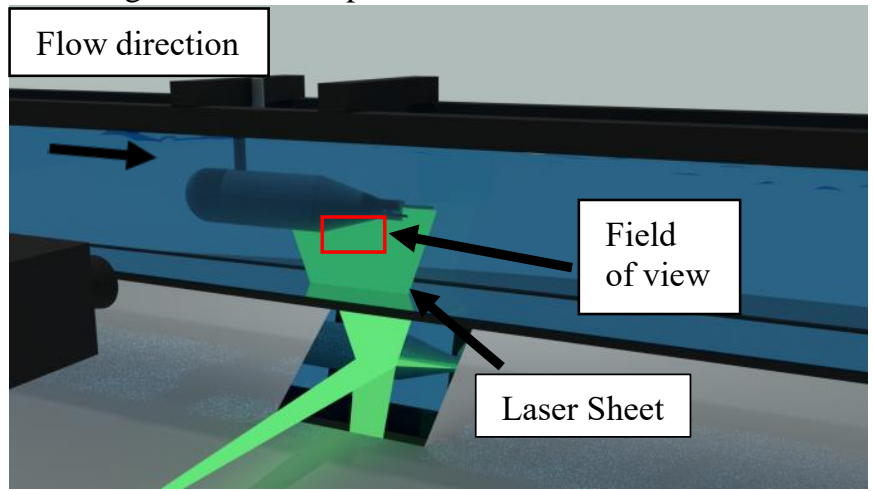

could be made on the bottom side. The tunnel had a height and width of 10 inches. A schematic of the full setup is shown in Fig. 2.

Fig. 2: Diagram of the experimental setup depicting the vessel in the water tunnel, the laser sheet, and the field of view.

16 $^{\text {th }}$ LACCEI International Multi-Conference for Engineering, Education, and Technology: "Innovation in Education and Inclusion”, 19-21 July 2018, Lima, Peru. 


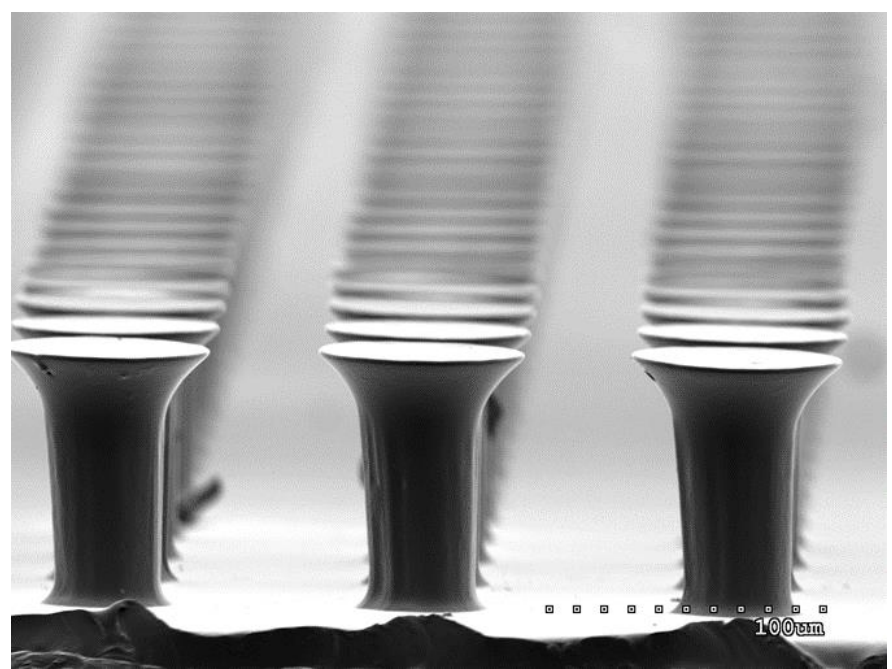

Fig. 3: Image of bio-inspired micro-fibers taken with an electron microscope.

The micro-fibers were constructed with a mushroom-like shape as shown in Fig. 3. The overhang around the pillar head makes the micro-fibers similar to shark denticles while allowing for easier fabrication in large quantities through the use of a molding procedure. The diameter of the circular top face of a pillar is $75 \mu \mathrm{m}$ while the diameter of the cylindrical stalk is $40 \mu \mathrm{m}$. The height of the diverging conical section is approximately $18 \mu \mathrm{m}$ from the top face to the start of the vertical pillar. The total height of each micro-fiber is $85 \mu \mathrm{m}$. The spacing from the center of one micro-fiber to center of the nearest micro-fiber is $120 \mu \mathrm{m}$. The micro-fibers are arranged in a square-packing pattern.

The flow field of the tapered section, where recirculation was expected, was evaluated using Particle Tracking Velocimetry (PTV). The laser used in the data acquisition had a width of between 1 to $2 \mathrm{~mm}$ and was projected from beneath the water channel creating a vertical sheet of continuous light. Images were captured at a rate of 125 frames per second at a resolution of 1280 by 1024 pixels. Calibration of this system resulted in 8 pixels $/ \mathrm{mm}$. The velocity fields were generated using a PIV-PTV hybrid algorithm with initial interrogation window of 96 square pixels and producing a result of 1999 velocity fields. These velocity fields where then time-averaged to depict the general activity of the flow.

\section{RESULTS AND DISCUSSION}

The average normalized velocity fields near the tapering end of the vessels reveal differences in flow around the microfiber coated vessel and the smooth coated vessel. The time averaged velocity fields have been evaluated using the perpendicular streamwise and vertical directions. The streamwise direction corresponds to the initial direction of the incoming flow. The vertical direction corresponds to the direction perpendicular to the streamwise direction that most directly approaches the external walls of the vessel.

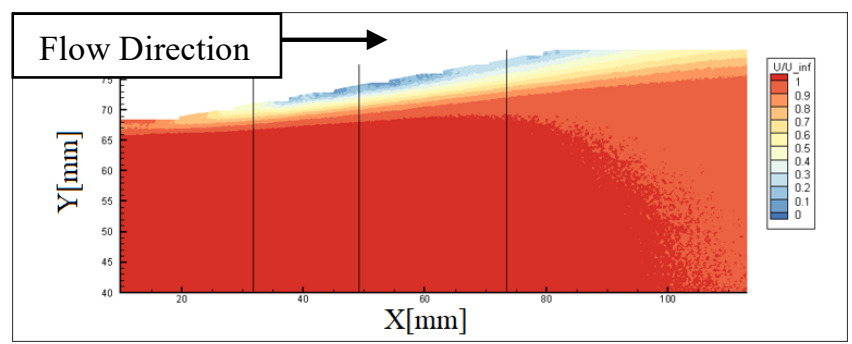

Fig. 4:Flow velocity with smooth coating in X-direction normalized to freestream $X$-direction velocity.

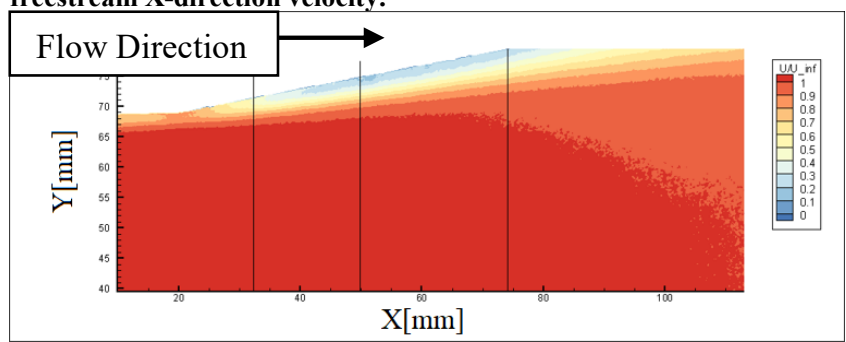

Fig. 5: Flow velocity with micro-fiber coating in X-direction normalized to freestream $\mathrm{X}$-direction velocity.

The comparison of Fig. 4 and Fig. 5 shows, most importantly, an increase in the flow velocity in the near-wall region of the micro-fiber coated vessel, represented by the lighter blue in Fig. 5. Since the conditions for flow separation require flow to stop at some point along the surface this increase in velocity would indicate a departure from this condition and suggest better flow conditions with the use of the micro-fiber coating.

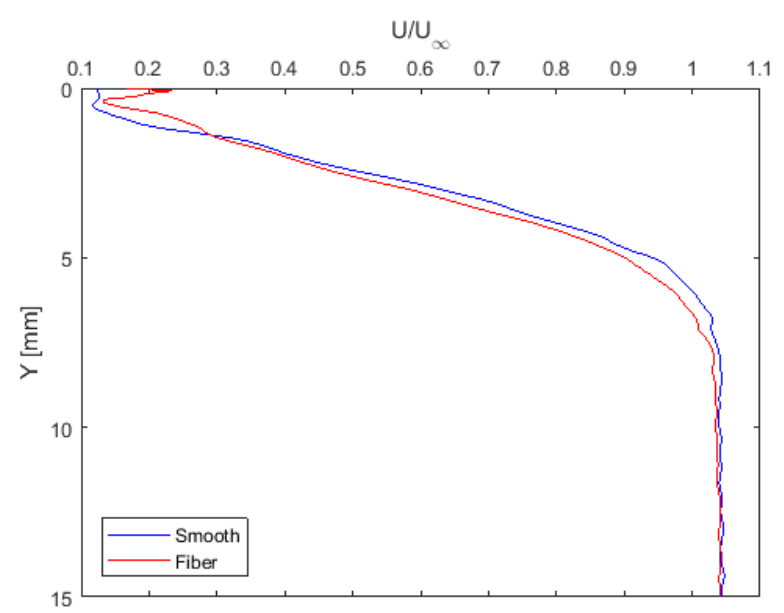

Fig. 6: Comparison of normalized $\mathrm{X}$-direction velocities for vessels with smooth and micro-fiber coatings. Note: $Y$ position is distance from vessel wall. 


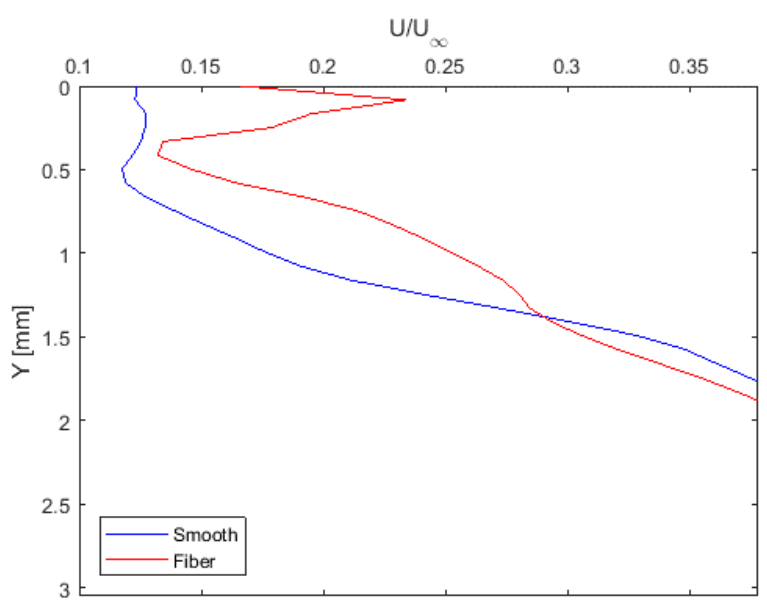

Fig. 7: Near wall comparison of normalized $X$-direction velocities for vessels with smooth and micro-fiber coatings with respect to vertical distance from the vessel wall. Note: $Y$ position is distance from vessel wall.

Fig. 6 shows an overview of the velocity profiles for both the micro-fiber and smooth vessels as evaluated at the middle vertical line of Fig. 4 and Fig. 5. For much of the profile, the differences in the normalized streamwise velocity are small and do not give preference to either micro-fiber or smooth coating having a higher velocity. Fig. 7 is a close-up view of the near wall velocity profile in the streamwise direction. An increase in the velocity near the wall can be observed.

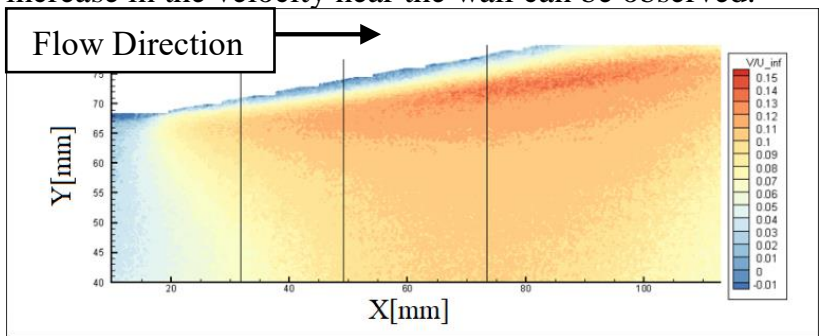

Fig. 8: Flow velocity with smooth coating in Y-direction normalized to freestream $\mathrm{X}$-direction velocity.

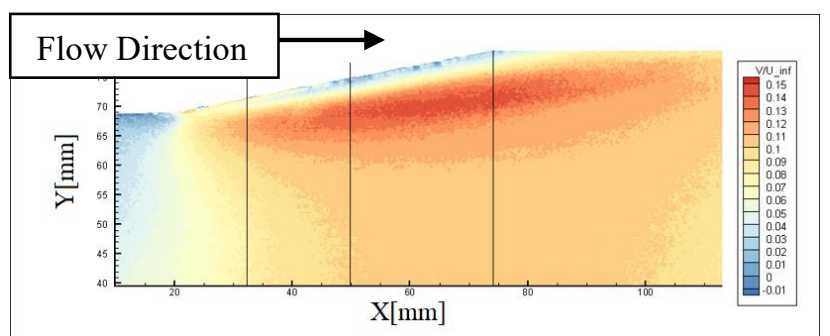

Fig. 9: Flow velocity with micro-fiber coating in Y-direction normalized to freestream $\mathrm{X}$-direction velocity.

The vertical velocity component of the experiments can be seen in Fig. 8 and Fig. 9, normalized by the same streamwise velocity that was used earlier. These velocity fields make it clear that in the midrange between the wall and the far field there is an increase in the vertical component from the vessel coated with the micro-fibers. What is less obvious is the increase in the vertical component next to the wall.

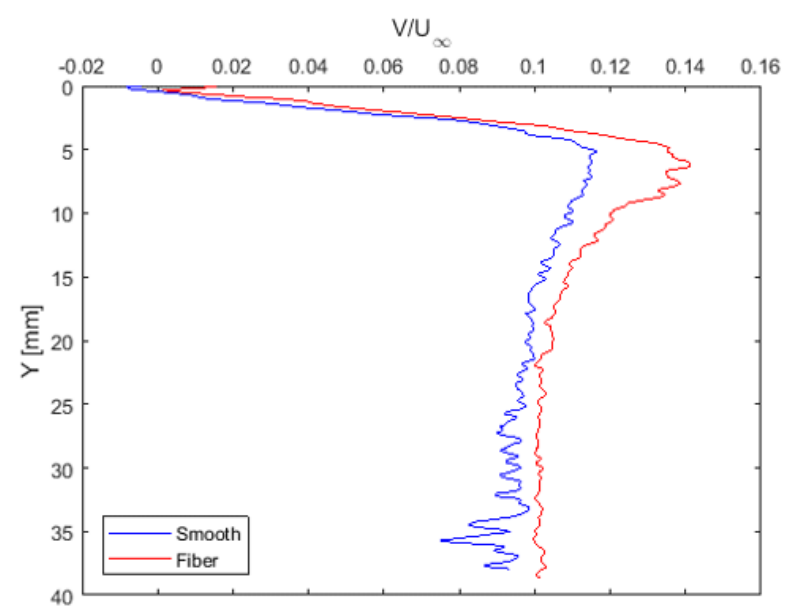

Fig. 10: Comparison of normalized $Y$-direction velocities for vessels with smooth and micro-fiber coatings with respect to vertical distance from the vessel wall. Note: $Y$ position is distance from vessel wall.

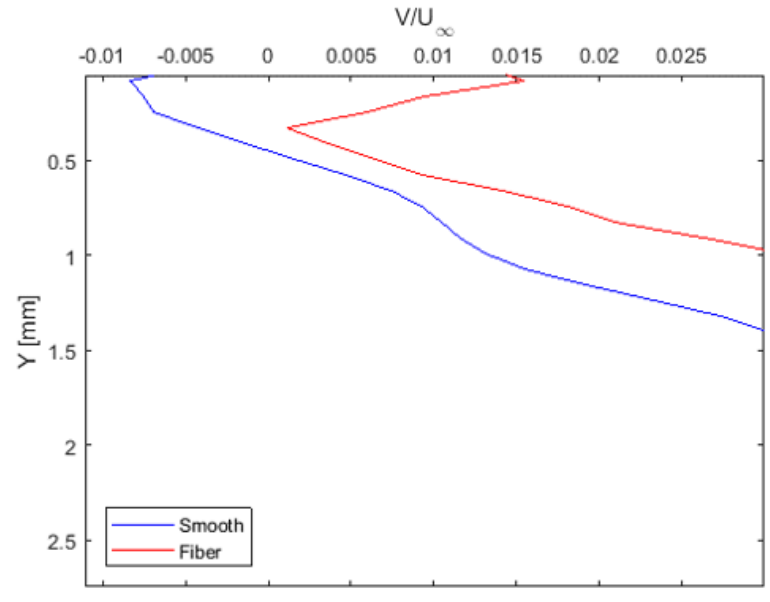

Fig. 11: Near wall comparison of normalized Y-direction velocities for vessels with smooth and micro-fiber coatings with respect to vertical distance from the vessel wall. Note: $Y$ position is distance from vessel wall.

Fig. 10 presents the velocity profile of the vertical velocity as evaluated at the middle vertical line of Fig. 8 and Fig. 9. There is a consistent trend of increased vertical velocity toward the vessel with an increase in the peak at approximately $5 \mathrm{~mm}$ from the vessel wall. This increase in the peak vertical velocity indicates that the flow is flowing toward the wall of the vessel with less likelihood of deviation. When looking at the velocity component near the wall, as shown in Fig. 11, a similar phenomenon as seen in the streamwise component is found. Further investigation into this heightened flow velocity near the wall and the short distance of decreasing velocity may give insights into the mechanism by which these bioinspired micro-fibers reduce flow separation.

\section{UNCERTAINTY ANALYSIS}

In order to lend perspective to the likely error in the measurements presented by the combination of PIV and PTV 
as described earlier a common error estimate for a 32-pixel by 32-pixel interrogation window PIV analysis is used. While this comparison is not exact it should lend some insight into the expected error. According to references [9] and [10] the typical bias for such a region is approximately 0.1 pixels. Using this approximation and the specifications for the experimental setup in this study the expected normalized error is determined to approximately 0.011 . This error expectation would suggest that the measurements found for the $\mathrm{x}$-direction near the wall are of great enough difference that the conclusions should not be attributed to PIV/PTV measurement error. The same, however, cannot be said for the vertical direction. While some of the measurements exceed the estimated error the conclusions from these graphs should be treated with more suspicion.

\section{CONCLUSION}

Experimental measurements using PTV have been made on a vessel coated with a biologically inspired surface and compared with a vessel coated with a smooth surface made of the same material. The differences in the flow fields show that the micro-fiber coating entices the flow to follow the vessel wall more closely in a region where the wall diverges from the flow's previous path. This flow also exhibits an increase in velocity along the wall of the vessel. Separation was not observed in this experiment to a degree that would allow for a definitive comparison of the micro-fiber coated vessel and the smooth coated vessel. As such the determination of separation reduction by the bio-inspired micro-fibers is difficult to assess and may be more easily interpreted under conditions of greater adverse pressure gradient. However, based on the other findings and the known causes of separation, the micro-fiber coating shows a manipulation of flow patterns that suggest flow separation could be delayed or reduced. Further investigation is needed to prove this possibility.

\section{REFERENCES}

[1] F. M. White, Fluid Mechanics, Seventh Edition, New York, NY: McGraw-Hill, 2011.

[2] S. Song and J. K. Eaton, "The effects of wall roughness on the separated flow over a smoothly contoured ramp," Experiments in Fluids, no. 33, p. 38-46, 2002.

[3] C. Baweja, R. Dhannarapu, U. Niroula and I. Prakash, "Analysis and Optimization of Dimpled Surface Modified for Wing Planforms," in 7th International Conference on Mechanical and Aerospace Engineering, 2016.

[4] E. M. Jones, "An Experimental Study Of Flow Seperation Over a Flat Plate With 2D Transverse Grooves," 2013.

[5] E. J. ANDERSON, P. S. MAcGILLIVRAY and M. E. DEMONT, "Scallop Shells Exhibit Optimization of Riblet Dimensions for Drag Reduction," The Biological Bulletin, no. 192, pp. 341-344., 1997.
[6] C. Xu, J. Wang, S. Luan, B. Qu and L. Jiang, "Analysis of Drag Reduction Mechanism of the Bionic Microscopic Riblets Surface," in 3rd International Conference on Biomedical Engineering and Informatics, 2010.

[7] L. Wen, J. C. Weaver and G. V. Lauder, "Biomimetic shark skin: design, fabrication and hydrodynamic function," The Journal of Experimental Biology, no. 217, pp. 1656-1666, 2014.

[8] H. B. Evans, A. M. Hamed, S. Gorumlu, A. Doosttalab, B. Aksak, L. P. Chamorro and L. Castillo, "Engineered bio-inspired coating for passive flow control," Proceedings of the National Academy of Sciences of the United States of America, 2017.

[9] A. Boomsma and F. Sotiropoulos, "Riblet drag reduction in mild adverse pressure gradients: A numerical investigation," International Journal of Heat and Fluid Flow, no. 56, pp. 251-260, 2015.

[10] J. Lee, Z. Zhang, S. Baek, S. Kim, D. Kim and K. Yong, "Bio-inspired dewetted surfaces based on $\mathrm{SiC} / \mathrm{Si}$ interlocked structures for enhanced-underwater stability and regenerative-drag reduction capability," Scientific Reports, 2016.

[11] Y. Luo, Y. Liu, J. Anderson, X. Li and Y. Li, "Improvement of water-repellent and hydrodynamic drag reduction properties on bio-inspired surface and exploring sharkskin effect mechanism," Applied Physics $A$, no. 120 , p. $369-377,2015$.

16 $^{\text {th }}$ LACCEI International Multi-Conference for Engineering, Education, and Technology: "Innovation in Education and 